\title{
COMPORTAMIENTO EN EL TIEMPO DE CASOS DE HIDATIDOSIS EN EL DEPARTAMENTO DE JUNIN DURANTE 1991 - 2002
}

\section{BEHAVIOR IN THE TIME OF CASES OF HIDATIDOSIS IN THE DEPARTMENT OF JUNIN DURING 1991 - 2002}

\author{
Luís Zúñiga Villacresis Gutiérrez', Ángela Ávila Chirinos², \\ Carmen Samaniego Durand ${ }^{2}$, Lola Salazar Sandoval ${ }^{2}$
}

\section{RESUMEN}

La hidatidosis humana es una enfermedad crónica que causa serios problemas de salud en muchas regiones del mundo en desarrollo, su importancia en la salud pública está relacionada no solo con el elevado índice de mortalidad humana, sino también con las pérdidas por rendimiento laboral, gastos de hospitalización, intervenciones e incapacidades. El objetivo fue determinar la frecuencia de casos de hidatidosis de acuerdo a sus variables demográficas, geográficas y a través del tiempo en el departamento de Junín durante los años 1991 al 2002. Estudio fue de tipo descriptivo, longitudinal y retrospectivo de casos de hidatidosis que fueron atendidos en los diferentes hospitales del departamento de Junín. Se realizó la revisión de los archivos de las diferentes instituciones de salud e identificación de pacientes con diagnóstico de hidatidosis atendidos por consulta externa u hospitalización y con examen radiológico, serológico o quirúrgico positivo para esta enfermedad. Se hallaron 1100 casos nuevos en su mayoría procedentes de la provincia de Huancayo (40,0\%), seguido de Yauli $(18,4$ $\%)$, Jauja $(7,7 \%)$ y Chupaca $(7,5 \%)$. Predominó en el género femenino $(56.7 \%$ ) y en los grupos de edad de la segunda y cuarta década de la vida. El 82,5\% fueron de de procedencia urbana. El 44,9 \% solo habían cursado primaria y secundaria incompleta. La localización pulmonar $(37,4 \%)$ predominó sobre las demás localizaciones. La tasa de mortalidad fue de 1,9\%. El departamento de Junín presenta una elevada casuística de hidatidosis humana por lo que urge implementar un programa de prevención y control inmediatos.

Palabras clave: Quiste hidatídico, equinoccus granulosus, hidatidosis.

\begin{abstract}
The human hidatidosis is a chronic illness that causes serious problems of health in many regions of the world in development, its importance in the health it not publishes this related one alone with the high index of human mortality, but also with the losses for labor yield, expenses of hospitalization, interventions and inabilities. The objective was to determine the frequency of cases of hidatidosis according to their demographic, geographical variables and through the time in the department of Junín during the years 1991 at the 2002. The study it was of descriptive type, longitudinal and retrospective of cases of hidatidosis that were assisted in the different hospitals of the Department of Junín. One carries out the revision of the files of the different Institutions of Health and identification of patient with I diagnose of hidatidosis assisted by external consultation or hospitalization and with radiological exam, serologico or surgical positive for this illness. They were 1100 new cases in their majority coming from the county of Huancayo $(40,0 \%)$, followed by Yauli $(18,4 \%)$, Jauja $(7,7 \%)$ and Chupaca $(7,5 \%)$. I Prevail in the I generate feminine $(56,7 \%)$ and in the groups of age of second o'clock and quarter decade of the life. $82,5 \%$ was of of urban origin. $44,9 \%$ alone had studied primary and secondary incomplete. The lung localization $(37,4 \%)$ I prevail on the other localizations. The rate of mortality was of $1,9 \%$. The department of Junín presents a high casuistry of human hidatidosis for what urges to implement a program of prevention and immediate control.
\end{abstract}

Key words: Cyst hidatídico, equinoccus granulosus, hidatidosis)

\footnotetext{
'Facultad de Medicina Humana. Universidad Nacional del Centro del Perú.
}

${ }^{2}$ Facultad de Enfermería. Universidad Nacional del Centro del Perú. 


\section{INTRODUCCIÓN}

La hidatidosis humana es una enfermedad crónica que causa serios problemas de salud en muchas regiones del mundo en desarrollo, su importancia en la salud pública está relacionada no solo con el elevado índice de mortalidad humana, sino también con las pérdidas por rendimiento laboral, gastos de hospitalización, intervenciones e incapacidades.

En el Perú esta zoonosis afecta principalmente a las regiones ganaderas de la Sierra Central y la Sierra del Sur, afirmación que se corrobora con los siguientes estudios: Pérez Palma (1) encontró que la prevalencia de Hidatidosis en la comunidad de Chachayllo, Junín fue del 10,8 \% demostrando que la prevalencia de la Hidatidosis en la Sierra Central del Perú es una de las más altas a nivel latinoamericano y mundial. Ramón (2) determinó que la prevalencia en ovinos fue de $81,04 \%$ en la SAIS Tupac Amaru Ltda. No 1, Junín, Zúñiga (3) encontró que el mayor índice de hidatidosis animal y humana se ubica en las localidades pertenecientes a la provincia de Huancayo, Junín.

El objetivo de la investigación fue determinar la frecuencia de casos de hidatidosis de acuerdo a sus variables demográficas, geográficas y a través del tiempo en el departamento de Junín durante los años 1991 al 2002.

\section{MATERIAL Y MÉTODOS}

\section{Diseño de la Investigación}

El estudio es longitudinal, descriptivo y retrospectivo de casos de hidatidosis que fueron atendidos en los diferentes hospitales del departamento de Junín durante los años 1991 al 2002.

\section{Universo y Muestra}

\section{a. Universo:}

Integrado por todos los pacientes atendidos por consulta externa hospitalizados y/o derivados en los diferentes hospitales del departamento de Junín durante los años 1991-2002.

b. Muestra:

Integrada por todos los pacientes considerados casos nuevos de hidatidosis (pulmonar, hepática u otra localización) con examen radiológico,

serológico y/o quirúrgico positivo, Tathla 2. Distribución de pacientes por artecedente epiderniológico. atendidos por consulta externa, hospitalizados $\mathrm{y} / \mathrm{o}$ derivados en los diferentes hospitales del departamento de Junín y clínicas privadas durante los años 1991-2002 y que tenga residencia en dicha zona de por lo menos de un año.

Procedimiento y Recolección de Datos Instrumentos para la recolección de datos: La información de las historias clínicas se recolectó a través de una ficha.
Metodología utilizada para el procesamiento de datos:

La información registrada fue agrupada para su descripción en varias categorías: año de diagnostico, establecimiento de salud, servicio de atención, edad, género, procedencia, tiempo en lugar de procedencia, grado de instrucción, ocupación actual, crianza de perro, crianza de otros animales, familiares $\mathrm{y} / \mathrm{o}$ vecinos con esta enfermedad, serología (Westen Blot, Arco V, HAl, Elisa), radiología (radiografía, ecografía, TAC), hallazgos quirúrgicos, estancia hospitalaria, condición al egreso hospitalario y lugar de transferencia. Los resultados de la ficha de recolección de datos fueron tabulados y se analizaron en base a frecuencias, porcentajes, promedio, valores mínimos y máximos en el programa de SPSS versión 11.0

\section{RESULTADOS}

Durante los doce años de estudio se registraron en el departamento de Junín 1,100 casos nuevos de Tabla 1. Distribución de frecuencias por año de diagnóstico.

\begin{tabular}{|c|c|c|c|}
\hline Año de diagnóstico & Frecuencia & Porcentaje & Porcentaje acumulado \\
\hline 1991 & 31 & 2,8 & 2,8 \\
\hline 1992 & 34 & 3,1 & 5,9 \\
\hline 1003 & 56 & 6,0 & 10,0 \\
\hline 1994 & 62 & 6,8 & 18,5 \\
\hline 1995 & 130 & 1198 & 28,4 \\
\hline 1896 & 83 & 7,5 & 35,8 \\
\hline 1897 & 127 & 11,5 & 47,5 \\
\hline 1998 & 101 & 8,2 & 50,0 \\
\hline 1999 & 105 & 9,5 & $\infty, 2$ \\
\hline 2000 & 130 & 11,8 & 78,0 \\
\hline 2001 & 114 & 10,4 & 88,4 \\
\hline 2002 & 128 & 11,8 & 100,0 \\
\hline Total & 1100 & 100,0 & \\
\hline
\end{tabular}

hidatidosis humana, confirmados por diagnóstico, serológico y/o quirúrgico.

Al observar los valores obtenidos de los casos nuevos de hidatidosis por año de diagnostico (tabla 1), se puede apreciar el predominio de la casuística entre los años 1995 al 2002 que corresponde a 918 (83,5 \%) casos y el mayor numero de casos se hallaron en los establecimientos del Ministerio de Salud y clínicas privadas $52,7 \%$ (588 pacientes), siendo atendidos 
en su mayoría en los servicios de cirugía general $(65,9 \%)$ y cirugía pediátrica $(12,0 \%)$.

En valores absolutos y porcentuales la hidatidosis humana adquiere mayor importancia en las provincias de Huancayo con $440(40,0 \%)$ casos, Yauli $202(18,4 \%)$ casos, Jauja $85(7,7$ $\%)$ y Chupaca $82(7,5 \%)$ casos. Estas cuatro provincias representan $73,6 \%$ de los casos diagnóstico ocurridos en el departamento de Junín en el periodo de 1991-2002. La mayoría de los casos fueron estudiantes 403 (36,6\%), y las amas de casa, 253 (23,0\%), Agricultores y/o ganaderos constituyeron $84(8,3 \%)$ casos.

Con respecto a las variables de antecedentes epidemiológicos encontramos que 581 $(52,8 \%)$ casos habían criado perros alguna vez en su vida, $230(20,9 \%)$ casos habían criado otros animales tales como ovino, porcino, caprino, vacuno, etc. y 47 (4,3\%) casos manifestaron tener familiares 0 vecinos con antecedentes de hidatidosis (Tabla 2).

Con respecto al diagnostico por imágenes obtuvimos: $664(60,4 \%)$ casos presentaron imagen radiográfica sugestiva de hidatidosis, 727 (66,1 \%)

Tahla 3. Distribución de pacientes por diagnóstico de imãyen.

\begin{tabular}{|c|c|c|c|c|c|c|}
\hline \multirow[t]{2}{*}{ Irragen } & \multicolumn{2}{|c|}{ Radcolaf; } & \multicolumn{2}{|c|}{ Eicgiafa } & \multicolumn{2}{|c|}{ TAC } \\
\hline & $\mathbf{N}^{\mathbf{n}}$ & $\mathrm{ng}$ & H' & 3 & N & $q_{h}$ \\
\hline Gugestiva de hidatidcsis & 664 & 50,4 & 727 & 00,1 & 14 & 1,3 \\
\hline No slgestlra ce hidallyosls & 436 & 31,6 & $3: 3$ & $33 \mathrm{y}$ & $1 J \varepsilon \varepsilon$ & $\lfloor 8,7$ \\
\hline Tulal & 1100 & $10: \pi, n$ & IInn & 100,0 & 1100 & $100, \Gamma$ \\
\hline
\end{tabular}

casos presentaron imagen topográfica axial computarizada (TAC) sugestiva de hidatidosis (Tabla 3).

Por diagnostico prequirúrgico se halló que 494 $(44,9 \%)$ casos presentaron quistes hidatídico a nivel pulmonar, 357 (32,5\%) casos presentaron quistes a nivel hepático y 249 (22,6\%) casos con localización mixta (pulmonar más hepático) y otras

Tabla 4. Distribución de pacientes por localización de quiste en diagnóstico post quirúrgice en órganos mâs importantes.

\begin{tabular}{|c|c|c|c|}
\hline Viagnóetıco post quirürgıco & Frecuentia & Porcentaje & $\begin{array}{l}\text { Poreentaje } \\
\text { acumulado }\end{array}$ \\
\hline 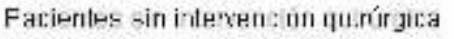 & 214 & 195 & 19.5 \\
\hline Fulmorar b latera & 45 & 4,1 & 23.5 \\
\hline Fuimor ar quuierdo & 152 & 13.8 & 37.4 \\
\hline Fulmorar $\mathrm{d} \rightarrow \mathrm{r}-\mathrm{r}$, 0 & 215 & 19,5 & 569 \\
\hline Hevalu & 331 & 30,1 & 87,6 \\
\hline Misto & 43 & 13,7 & 100,0 \\
\hline TOTA & 1100 & $100, \mathrm{~L}$ & \\
\hline
\end{tabular}

localizaciones.

Posterior a la intervención quirúrgica se encontró que $412(37,4 \%)$ casos presentaron quiste hidatídico a nivel pulmonar, $331 \quad(30,1 \%)$ a nivel letalidad especifica para hidatidosis.

\begin{tabular}{|c|c|c|c|c|}
\hline $\begin{array}{l}\mu^{0} \text { de casos } \\
\text { nuevos }\end{array}$ & $\begin{array}{l}\text { Población } \\
\text { Junin }\end{array}$ & $\mathrm{H}^{\circ}$ de fallecidos & $\begin{array}{l}\text { Mortalidad por } \\
100000 \\
\text { habitantes }\end{array}$ & Letalidad \\
\hline 31 & 1079426 & 1 & 0,09 & 3,2 \\
\hline 34 & 1095034 & 0 & 0,0 & 0,0 \\
\hline 55 & 1100201 & 1 & 0,09 & 1,8 \\
\hline 62 & 1125250 & 0. & 0,0 & 0,0 \\
\hline 130 & 1140505 & 1 & 0,09 & 0,8 \\
\hline 83 & 1156059 & 3 & 0,26 & 3,6 \\
\hline 127 & 1171698 & 3 & 0,26 & 2,4 \\
\hline 101 & 1187279 & 2 & 0.17 & 2,0 \\
\hline 105 & 1202663 & 1 & 0,08 & 1,0 \\
\hline 130 & 1217708 & 3 & 0,25 & 2,3 \\
\hline 114 & 1232343 & 0 & 0,0 & 0,0 \\
\hline 128 & 1246663 & 6 & 0,48 & 4,7 \\
\hline 1100 & & 21 & & 1,9 \\
\hline
\end{tabular}

hepático y $143(13,0 \%)$ con localización mixta (pulmonar más hepático y otras localizaciones), (Tabla 4).

Por hallazgo quirúrgico encontramos que 56,6 \% presentaron quistes entre 11 a $20 \mathrm{~cm}$ y el $34,8 \%$ entre 5 a $10 \mathrm{~cm}$. El tamaño promedio de quiste hallado por cirugía fue de $14 \mathrm{~cm}$, el tamaño mínimo fue de $2 \mathrm{~cm}$ y el máximo de $50 \mathrm{~cm}$.

El tiempo de estancia hospitalaria que observamos fue: el 40,0 \% fueron hospitalizados entre 8 a 14 días y el $24,5 \%$ tuvieron una estancia entre 15 y 21 días, siendo el valor promedio de días de estancia hospitalaria de 15 , la estancia hospitalaria mínima fue de 1 día y la máxima de 120 días.

En el periodo de estudio la mortalidad más elevada se halló en el año 2002 y la letalidad entre los doce años de estudio fue del 1,9\% (Tabla 5).

\section{DISCUSION}

En el periodo comprendido entre los años 1991 al 2002 se hallaron 1100 casos nuevos de hidatidosis humana en el departamento de Junín, confirmados por diagnostico radiológico, serológico y/o hallazgos quirúrgicos, cuya casuística se obtuvo revisando los archivos de las diferentes instituciones del Ministerio de Salud, instituciones de seguro social, instituciones de las fuerzas policiales y clínicas privadas, estudio nunca realizado anteriormente, (Tabla 2)

La mayor casuística se halló en los ocho 
últimos años del periodo de estudio (83,5\%), durante las cuatro primeros años se produjo una perdida de información producto de una baja capacidad de almacenamiento de las historias clínicas y/o traslado de las mismas al archivo pasivo realizado periódicamente en los diferentes establecimiento de salud. Los resultados de los últimos 8 años muestra una tendencia que el problema continua en vez de disminuir e incluso para el año 2002 se observa una mayor tasa de mortalidad $(0,48$ por 100000 habitantes $)$ y letalidad (4,7\%).(Tabla 5) De todas maneras este dato esta subestimado por lo indicado anteriormente y porque un gran número de casos no se llegan a diagnosticar sea por la ausencia de sintomatología, por los escasos recursos económicos que limita al paciente a acercarse a instituciones de salud o por la difícil accesibilidad geográfica y/o gran distancia de su vivienda hacia los, mismos.

La distribución por genero en los casos nuevos muestra un predominio de la hidatidosis en las mujeres $(56,7 \%)$, al analizar la distribución por edad de los 1100 casos nuevos de hidatidosis humana los grupos más afectados fueron de 10-14 y de 15-20 años que representan 294 (26,8 \%) casos, (Tabla 4).

Tomando en consideración los resultados expuestos se puede concluir que la hidatidosis es una enfermedad que afecta a personas de cualquier género y edad pero fundamentalmente a individuos en plena capacidad productiva.

Respecto a la distribución por procedencia de los casos nuevos de hidatidosis humana se registró que $951(86,5 \%)$ casos correspondían al departamento de Junín, 92 (8,4 \%) al departamento de Huancavelica, $377(3,4 \%)$ al departamento de Cerro de Pasco y 14 (1,3\%) al departamento de Lima.

En el departamento de Junín la mayor casuística se registró en las provincias de Huancayo (40,0\%), Yauli (1 8,4 \%), Jauja (7,7 \%) y Chupaca (7,5\%). En la provincia de Huancayo los distritos que presentaron mayor porcentaje estuvieron representados en primer lugar por el El Tambo $(11,7 \%)$ seguido de Huancayo (10,5 \%), Chilca $(7,5$ $\%)$, Huayucahi (3,0 \%), Pilcomayo (1,2\%), Huancan $(1,0 \%)$ y finalmente Sapallanga $(0,7 \%)$. Resultados que con respecto a la provincia de Huancayo, Jauja y Chupaca (Tabla 5). En consecuencia Huancayo como provincia presenta una elevada frecuencia de casuística de hidatidosis humana. Casuística se mantiene probablemente porque no se modifican los hábitos y conductas perniciosas que facilitan la infección de animales y del mismo hombre, pues de continua erróneamente con la alimentación de los perros con vísceras crudas infectadas con quistes hidatídicos obtenidos en su mayoría en las matanzas familiares o clandestinas, no se realiza una desparasitación canina sistémica, existe un inadecuado control en la inspección y decomiso de vísceras infectadas a nivel de camales impidiendo romper el ciclo evolutivo del Equinococus granulosus y falta de orientación y sensibilización de la población, lo que conlleva a un aumento de la enfermedad tanto a nivel de Huancayo como de todo el departamento .

La distribución por ocupación de los casos nuevos reveló que entre estudiantes se presentó el mayor porcentaje de casos $(39,7 \%)$ seguidos de las amas de casa $(25,0 \%$ ). Solo el $8,3 \%$ manifestaron estar vinculados a labores agrícolas y/o ganaderas.

El mayor porcentaje registrado en las ocupaciones mencionadas anteriormente tiene su posible explicación en la relación existente entre el perro y su propietario, ya que en esta zona y muchas otras el perro no es necesariamente un animal que sea utilizado en forma preferencial en albores relacionadas con pastoreo, y es así como no estaría en contacto permanente con el agricultor sino por el contrario es utilizado casi exclusivamente en labores de vigilancia del hogar y como compañero de juego siendo de este modo las amas de casa y los estudiantes quienes tienen una relación mas estrecha con este animal; también hay que reconocer que la hidatidosis es una enfermedad de evolución lenta, por lo que muchas veces las estadísticas sobre la ocupación actual del paciente no es muy relevante, ya que en el pasado pudieron haber desarrollado actividades que significaron un mayor riesgo de exposición ( $40 \%$ ).

En la distribución de pacientes por antecedentes epidemiológicos el 52,8\% manifestó haber criado perro alguna vez en su vida, el $20,9 \%$ criaba otros animales tales como: ovinos, porcinos, caprinos, vacunos, etc. y un mínimo porcentaje $(4,3 \%)$ refirió haber tenido familiares $y / o$ vecinos con hidatidosis. Estos datos no son muy contributarios porque en gran numero de casos no estuvo consignado en la historia clínica, representando perdida de información, así tenemos un mínimo de numero de casos con familiares y/o vecino con esta patología, conociendo que casos familiares se dan con más frecuencia, sin embargo muchos pasan inadvertidos por no realizar un despistaje adecuado, que aunque pueda parecer costoso, debería indicarse o sugerirse en aquellos familiares que hallan estado expuestos a los mismos factores de riesgo.

En los hallazgos post- quirúrgicos encontramos que la localización pulmonar fue la más frecuente $(37,4$ $\%)$, en segundo lugar tenemos la localización hepática ( $30,1 \%$ ) y en tercer lugar la localización mixta ( pulmonar más hepático y otras localizaciones) (13,0 \%) . A nivel pulmonar 
predomino el pulmón derecho (19,5\%), (Tabla 4).

Es importante destacar que en la hidatidosis, como en toda enfermedad de evolución prolongada la determinación de la tasa de mortalidad y letalidad es difícil; en efecto, solo se conoce el numero de individuos que fallecen por hidatidosis por año durante su estadía en el hospital desconociéndose cuantos mueran después del egreso los cuales no parecen consignados en las estadísticas hospitalarias Entre las causas de muerte de mayor frecuencia que se hallaron fueron insuficiencia respiratoria y shock anafiláctico.

\section{REFERENCIAS BIBLIOGRAFICAS}

1. Pérez Palma R. Prevalencia de Hidatidosis Humana en una Comunidad de la Sierra Central del Perú. (Tesis de optar el título de Médico). Lima: Universidad Nacional Mayor de San Marcos; 1998.

2. Ramón Rodríguez CM, Prevalencia y repercusión económica de hidatidosis y prevalencia de equinococosis en la SAIS Tupac Amaru Ltda. $N^{\circ}$ 1 (Tesis para optar el titulo de Ingeniero Zootecnista). Lima: Universidad Nacional Mayor de San Marcos; 1998.

3. Zúñiga R. La Hidatidosis en Huancayo, aspectos epidemiológicos y quirúrgicos. (Tesis doctoral). Lima: Universidad Nacional Mayor de San Marcos; 1976.

4. Uceda J. Hidatidosis en la Oroya Junín, estudio epidemiológico, nosográfico y tratamiento quirúrgico. (Tesis para optar el grado de Bachiller en Medicina). Lima: Universidad Nacional Mayor de San Marcos; 1965.

5. Castro R. Contribución al estudio de echinoccosis en la Provincia de Tarma del departamento de Junín. (Tesis). Lima: Universidad Nacional Mayor de San Marcos; 1961.

E-mail: enfermera_aach7@yahoo.com 